\title{
Eficiência técnica da produção agrícola dos países da América Latina e do Caribe
}

\author{
Technical efficiency of the agricultural production in Latin America and \\ Caribbean countries
}

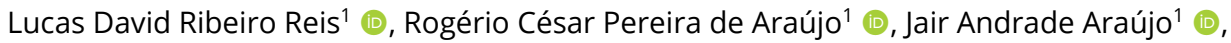 \\ João Ricardo Ferreira de Lima² (1) \\ ${ }^{1}$ Programa de Pós-graduação em Economia Rural (PPGER), Universidade Federal do Ceará (UFC), Fortaleza (CE), Brasil. E-mails: \\ lucas.david@facape.br; rcpa@ufc.br; jaraujoce@gmail.com \\ ${ }^{2}$ Empresa Brasileira de Pesquisa Agropecuária (Embrapa Semiárido), Petrolina (PE), Brasil. E-mail: joao.ricardo@embrapa.br
}

\author{
Como citar: Reis, L. D. R., Araújo, R. C. P., Araújo, J. A., \& Lima, J. R. F. (2020). Eficiência técnica da produção agrícola \\ dos países da América Latina e do Caribe. Revista de Economia e Sociologia Rural, 58(4), e219416. \\ https://doi.org/10.1590/1806-9479.2020.219416
}

Resumo: Este artigo estuda a eficiência técnica e a produtividade total dos fatores (PTF) da agricultura dos países da América Latina e do Caribe. Para tanto, utilizaram-se a abordagem de fronteira estocástica de produção e o índice de produtividade de Malmquist. Os dados de valor da produção e a quantidade de insumos agrícolas abrangeram os anos de 1991 a 2012. Os resultados mostraram que todos os países apresentaram ineficiência técnica de produção agrícola ao longo do período. As variáveis que explicaram significativamente a ineficiência da produção agrícola foram o crédito e o consumo de energia na agricultura, o que representou uma inovação nessa temática. O índice de produtividade de Malmquist mostrou que não houve ganhos de produtividade total dos fatores.

Palavras-chave: agricultura, fronteira estocástica de produção, Índice de Malmquist.

\begin{abstract}
This article studies the technical efficiency and the total productivity of agricultural factors in the countries of Latin America and of Caribbean. For this, it was applied the stochastic production frontier approach and the Malmquist productivity index. The data used were the value of production and the quantity of agricultural inputs from the years 1991 to 2012. The results showed that all countries presented technical inefficiency of agricultural production over the period. The variables that significantly explained the inefficiency of agricultural production were credit and energy consumption in agriculture, which represented an innovation in this area. The Malmquist productivity index showed that there were no gains in total factor productivity.
\end{abstract}

Keywords: agriculture, stochastic frontier of production, Malmquist Index.

\section{Introdução}

O agronegócio é tido como a soma de todas as operações ao longo da cadeia agroalimentar, compreendendo o setor fornecedor de insumos, a produção em nível primário, o processamento e a distribuição. Segundo Hutchinson (2007), o setor agropecuário há muito tempo deixou os países da América Latina e do Caribe (ALC) dependentes dele, tanto como fonte de alimentos como também de divisas geradas pelas exportações. Mesmo com os recentes aumentos no setor de turismo nesses países, a agricultura é ainda muito importante, não apenas como fonte de receita, mas também pela geração de emprego, especialmente nas áreas rurais (Hutchinson, 2007).

A América Latina e o Caribe também são tidos como as maiores regiões exportadoras líquidas de alimentos do mundo (Ferreira et al., 2016). De acordo com indicadores do Inter-American Development Bank (IADB), a produção agrícola dos países pertencentes à América Latina deve 
apresentar crescimento de $80 \%$ até 2050, para atender a um esperado aumento na sua população, mais de 35\% no mesmo período (Inter-American Development Bank, 2014).

O Brasil é o país que apresenta a maior economia desse bloco, apresentando uma das maiores produções agrícolas do mundo, não somente em volume, mas também em produtividade (Ferreira et al., 2016). A área plantada de grãos brasileira cresceu $42 \%$ nos últimos 30 anos, ao passo que a produção teve crescimento de $228 \%$. Enquanto a área plantada avançou 17 milhões de hectares, a produção se expandiu em 133 milhões de toneladas, gerando um ganho de produtividade de 3,2\% ao ano. Se os demais países da América Latina tivessem os níveis de produtividade do Brasil, a área cultivada seria 17\% menor, economizando, assim, 8,2 milhões de hectares (Brasil, 2014).

A partir da década de 1960, a inclusão de novos pacotes tecnológicos, baseados na Revolução Verde, inseridos na agricultura da América do Sul, foi essencial para o aumento de produtividade dos países desse bloco. A exemplo dessas tecnologias, têm-se os adubos químicos e a maquinaria agrícola (Montoya, 2002).

A agropecuária não é importante somente na geração de emprego e renda, mas também na própria questão da segurança alimentar, como abordado em Gomes et al. (2013). O aumento da população faz com que a demanda por alimentos também aumente e, assim, com a quantidade de terra produzível cada vez mais limitante, a produtividade do setor agrícola deve ser cada vez mais elevada para atender às necessidades da população (Zúniga-González, 2011).

Assim, tendo em vista a grande importância da produtividade agrícola, este trabalho busca avaliar a eficiência técnica da agricultura na América Latina e no Caribe (ALC). Para isto, se recorrerá à análise de fronteira estocástica de produção, a qual permite estimar os escores de eficiência técnica dos países latino-americanos.

Além da eficiência técnica da agricultura, será analisada também, através do índice de Malmquist, a produtividade total dos fatores agrícolas. Este índice se sobressai aos demais, visto que pode ser decomposto em dois outros, que são: a variação do nível de eficiência e a mudança tecnológica. A vantagem de se utilizar tal método é que ele permite identificar se é a variação na eficiência técnica ou o progresso técnico que mais contribui para o crescimento da produtividade total dos fatores da agricultura nos países da América Latina e do Caribe.

O artigo, além desta introdução está dividido em mais quatro seções. Na seção 2, se encontra a revisão de literatura de trabalhos que estudaram a eficiência técnica agrícola na América Latina e no Caribe. Na terceira seção, apresenta-se a metodologia utilizada neste trabalho: a fronteira de produção estocástica, os testes de hipóteses, o índice de produtividade de Malmquist e a fonte de dados. Os resultados e discussões estão descritos na quarta seção. A seção cinco, por sua vez, faz as considerações finais do estudo.

\section{Revisão de Literatura}

Nesta seção, mostrar-se-á uma breve revisão acerca da eficiência técnica da agricultura e da agropecuária nos países da ALC.

\subsection{Eficiência técnica agrícola na América Latina}

Na literatura, existem trabalhos que tratam da questão da eficiência e da produtividade da agricultura na América Latina e no Caribe que se diferenciam quanto aos países analisados, ao período de abrangência e aos métodos e variáveis utilizados. Alguns estudos focaram apenas nos países do Caribe (Hutchinson, 2007; Zúniga-González, 2011), enquanto outros focaram nos países da América Latina e do Caribe (Ludena, 2010; Ferreira et al., 2016), ou da América do Sul (Carvalho \& Marinho, 2003; Trindade \& Fulginiti, 2015; Helfand et al., 2015). Vale também destacar os trabalhos que incluíram as variáveis ambientais (e.g. emissões de $\mathrm{CO}_{2}$ ) em seus modelos no intuito de medir a PTF (Ebata, 2011; Moreno-Moreno et al., 2017). A seguir, cada um desses trabalhos é apresentado brevemente.

Hutchinson (2007) analisou a eficiência técnica agrícola de 11 países do Caribe, entre 1961 e 2000, calculada pelo método Data Envelopment Analysis (DEA) e pelo índice de produtividade de Malmquist. Para avaliar as mudanças na produtividade dos países, o autor 
dividiu a amostra em dois períodos: 1961-1981 e 1981-2000. Constatou que, no primeiro período, apenas cinco países apresentaram ganhos de PTF, enquanto que, no segundo período, esse número saltou para oito países. Em ambos os períodos, os ganhos de PTF somente ocorreram devido à expansão da fronteira tecnológica agrícola (progresso técnico).

Zúniga-González (2011), utilizando também o DEA e o índice de produtividade de Malmquist, analisou a eficiência do setor agrícola de 14 países do Caribe, no período de 1979 a 2008. Os resultados mostraram um crescimento anual da PTF de 1,5\%, em que a mudança de eficiência técnica contribuiu com $0,1 \%$ ao ano e a mudança de fronteira (tecnológica) proporcionou 1,4\%. Os resultados de Zúniga-González (2011) corroboraram com os de Hutchinson (2007).

Ludena (2010), também utilizando os métodos dos dois estudos apresentados, analisou a eficiência do setor agropecuário nos países da ALC, no período de 1961 a 2007. Os resultados demonstraram que os países investigados apresentaram crescimento da produtividade total dos fatores, especificamente nas últimas duas décadas do período analisado. Essas mudanças foram atribuídas às melhorias de eficiência e à introdução de novas tecnologias na agricultura.

Carvalho \& Marinho (2003) analisaram a produtividade total dos fatores da produção agrícola na América do Sul, separando-os entre países membros e não membros do MERCOSUL. Utilizando o método DEA e o Índice de Malmquist, observaram perdas de eficiência técnica, em média, entre os países, e acréscimos na produtividade total dos fatores, em grande parte, devidos a variações tecnológicas.

Trindade \& Fulginiti (2015) estimaram a produtividade agrícola na América do Sul, entre os anos de 1969 e 2009. Os autores concluíram que o aumento da produtividade agrícola foi ocasionado por inovações no setor. Além disso, eles mostraram que os gastos com educação e P\&D na agricultura são importantes para entender o desempenho agrícola entre os países desta região.

Ferreira et al. (2016), utilizando a fronteira estocástica de produção em painel, também analisaram a produtividade total dos fatores de 19 países da América Latina. Segundo os autores, a ineficiências técnica dos países investigados foi diminuída significativamente pela educação e pelo open market. Verificaram ainda que todos os países apresentaram variação da PTF positiva no período entre 1961 e 2010, ou seja, apresentaram crescimento do produto, assim como progresso técnico positivo.

Ebata (2011) analisou o processo produtividade na América Central e no Caribe entre os anos de 1976 e 2006. Este estudo, em particular, incluiu a variável de emissões de $\mathrm{CO}_{2}$ em sua análise, no intuito de capturar o efeito dos fatores da mudança climática na agricultura. Constatou-se que, nesse período, a produtividade agrícola aumentou quando as variáveis ambientais, como as emissões, foram omitidas. Contudo, quando as emissões $\mathrm{CO}_{2}$ foram adicionadas à análise, observou-se um ligeiro declínio no crescimento da produtividade agrícola entre 1992 e 2006.

Moreno-Moreno et al. (2017) analisaram a eficiência da produção agropecuária de 25 países da ALC com dados seccionais do ano de 2012. Utilizando a técnica DEA, compararam os resultados de três modelos, em que consideraram como produtos: a produção agropecuária no primeiro modelo; as emissões de $\mathrm{CO}_{2}$ no modelo 2, e a produção agropecuária e as emissões de $\mathrm{CO}_{2}$ no terceiro modelo. Os resultados mostraram que a prioridade dos países da ALC ainda é a eficiência técnica da produção agrícola e que os regulamentos de produção ambiental não têm sido suficientes para incentivar a redução das emissões de $\mathrm{CO}_{2}$ na agricultura.

Esses estudos mostram que os estudos de eficiência e produtividade na agricultura ampliam-se no sentido de incorporar nas análises as variáveis ambientais e avaliar seu efeito no desempenho produtivo do setor agropecuário. Nesse sentido, este artigo inova ao incorporar o consumo de energia como variável ambiental e fonte de ineficiência técnica na agropecuária da ALC. 


\section{Metodologia}

Nesta seção, mostrar-se-á o modelo econométrico de fronteira estocástica de produção e os testes estatísticos realizados. Em seguida, apresentam-se a função de produção utilizada no modelo empírico e o cálculo do índice de produtividade de Malmquist. Por último, descrevem-se as fontes de dados da pesquisa.

\subsection{Fronteira Estocástica de Produção}

Para o cálculo da eficiência técnica dos países analisados, propõe-se utilizar a metodologia de fronteira estocástica de produção (stochastic frontier of production - SFA) proposta por Aigner et al. (1977) e Meeusen \& Broeck (1977). Posteriormente, Battese \& Coelli (1995) possibilitaram estimar este modelo com dados estruturados em painel, no qual a ineficiência técnica de produção estava relacionada a um vetor de outras variáveis.

A função de produção para uma Unidade de Tomada de Decisão (UTD) ${ }^{1} i$ no ano $t$ é definida, conforme Battese \& Coelli (1995), como:

$y_{i t}=\exp \left(x_{i t} \beta+v_{i t}-u_{i t}\right)$,

Em que: $y_{i t}$ é o vetor de quantidades produzidas (outputs) pelo i-ésimo país no t-ésimo ano; $x_{i t}$ é o vetor de insumos (inputs) utilizados na produção pelo i-ésimo país no t-ésimo ano; e $\beta$ é o vetor de coeficientes a serem estimados.

A composição do termo de erro é dada por $v_{i t}$ e $u_{i t}$, que são tidos como independentes entre si (Coelli et al., 1998; Battese \& Coelli, 1995). O primeiro termo, $v_{i t}$, representa um conjunto de variáveis aleatórias assumidas como independentes e identicamente distribuídas, com distribuição normal de média zero e variância constante, iid $N \sim\left(0, \sigma_{v}^{2}\right)$. Esse termo captura os erros de medição, ruídos estatísticos e choques aleatórios fora do controle da UTD, e os efeitos das variáveis explicativas não especificadas na função de produção.

O segundo termo, $u_{i t}$, representa um conjunto de variáveis aleatórias não negativas (truncada em zero) que são responsáveis pela ineficiência da produção, podendo o mesmo assumir a distribuição normal truncada, a half-normal, a exponencial e a gamma (Coelli et al., 1998).

Battese \& Coelli (1995) buscaram explicar a eficiência técnica por meio de outras variáveis, sendo:

$u_{i t}=z_{i t} \delta+w_{i t}$,

Em que: $z$ é um vetor de variáveis explicativas da ineficiência da i-ésima firma no t-ésimo ano; $\delta$ é um vetor de parâmetros a serem estimados associados às variáveis $z_{i t}$; e $w_{i t}$ é uma variável aleatória que se comporta como um ruído branco (white noise), ou seja, $N \sim\left(0, \sigma^{2}\right)$. A ineficiência técnica, $u_{i t}$, como tendo uma distribuição normal truncada em zero, cuja média é igual a $z_{i t} \delta$. Os parâmetros $\delta^{\prime}$ S na Equação 2 e $\beta^{\prime}$ S em (1), são estimados simultaneamente via Máxima-Verossimilhança.

A eficiência técnica por orientação ao produto para o i-ésimo país no t-ésimo ano é dada pela razão entre o produto observado e o produto de fronteira. Uma vez que $u_{i t} \geq 0$, isso assegura que todas as observações estejam situadas na fronteira ou abaixo dela. Assim, a eficiência técnica é dada por (Coelli et al., 1998; Battese et al., 2004; O’Donnell et al., 2008):

\footnotetext{
1 O termo UTD é a tradução livre do termo DMU (Decision Making Unit), já que tal método pode ser aplicado não somente às firmas. O termo UTD refere-se a qualquer unidade tomadora de decisão que transforme insumos em produtos, podendo ser firmas, instituições, setores da economia, regiões ou como este trabalho, que estima a eficiência técnica da agricultura dos países da ALC.
} 


$$
E T_{i t}=\frac{y_{i t}}{\exp \left(x_{i t} \beta+v_{i t}\right)}=\frac{\exp \left(x_{i t} \beta+v_{i t}-u_{i t}\right)}{\exp \left(x_{i t} \beta+v_{i t}\right)}=\exp \left(-u_{i t}\right)
$$

Substituindo a Equação 2 na Equação 3, temos que a eficiência técnica de produção do i-ésimo país no t-ésimo ano é dada, segundo Battese \& Coelli (1995), por:

$E T_{i t}=\exp \left(-z_{i t} \delta-w_{i t}\right)$

Aigner et al. (1977) parametrizaram a função log-máxima verossimilhança, passando a ser expressa em termos da variância dos parâmetros: $\sigma^{2}=\sigma_{v}^{2}+\sigma_{u}^{2}$ e $\lambda^{2}=\sigma_{u}^{2} / \sigma_{v}^{2} \geq 0$. Se $\lambda=0$, a ineficiência técnica é inexistente e todos os desvios da fronteira são devidos aos ruídos estocásticos.

Battese \& Corra (1977) consideraram mais conveniente parametrizar a função de log-máxima verossimilhança em termos de $\sigma^{2}=\sigma_{v}^{2}+\sigma_{u}^{2}$ e $\gamma=\sigma_{u}^{2} / \sigma^{2}$. O parâmetro $\gamma$ encontra-se entre os valores zero e a unidade. Se $\gamma=0$, tem-se que todos os desvios à fronteira de produção devem-se a choques aleatórios; se $\gamma=1$, tem-se que todos os desvios são causados pela ineficiência técnica.

\subsection{Modelo Econométrico}

Neste estudo, primeiramente, o modelo foi estimado com as funções de produção Cobb-Douglas e Translog. De acordo com a razão de verossimilhança (LR), a função translog ajustou melhor os dados, embora esta não tenha rejeitado a hipótese de ausência de ineficiência, obtida ao se compararem os modelos OLS e SFA. Assim, a Cobb-Douglas foi selecionada pelo fato de ser uma função amplamente utilizada para estimar SFA e ter rejeitada a hipótese de ausência de ineficiência.

As variáveis que explicam a produção foram os insumos clássicos (terra, capital, trabalho e tempo). Essas variáveis também foram utilizadas nas funções de produção estimadas em Marinho \& Carvalho (2004), Marinho \& Bittencourt (2007), Araújo et al. (2014), Ferreira et al. (2016) e Araújo \& Araújo (2016).

A função de produção Cobb-Douglas proposta nesse estudo é expressa pela Equação 5, a seguir:

$\ln P R O D U C A O_{i t}=\beta_{0}+\beta_{1} \ln A R E A_{i t}+\beta_{2} \ln T R A B A L H O_{i t}+\beta_{3} \ln M A Q U I N A_{i t}+\beta_{4} T E M P O+v_{i t}-u_{i t}$,

Em que: In denota o logaritmo natural (i.e., o logaritmo na base $e$ ); PRODUCAO it é a quantidade produzida pela agricultura do país $i$ no período $t ; A R E A_{i t}$ é o fator terra do país $i$ no período $t$; $T_{R A B A L H O}$ it representa o fator trabalho do país $i$ no período $t$; MAQUINA $A_{i t}$ o número de máquinas agrícolas do país i no período t; TEMPO é uma variável de tendência, que captura os efeitos ao longo do tempo; $v_{i t}$ representa as perturbações aleatórias assumidas como independentes e identicamente distribuídas com distribuição normal de média zero e variância constante, iid $N \sim\left(0, \sigma_{v}^{2}\right)$; e $u_{i t}$ é a ineficiência técnica de produção.

As variáveis selecionadas para explicar a ineficiência técnica foram o montante de crédito agrícola e o consumo de energia no setor agropecuário. O acesso ao crédito, segundo Scherer \& Porsse (2017), está associado, tanto direta como indiretamente, à ampliação do sistema produtivo na agricultura. O crédito viabiliza, de forma direta, a aquisição de novos insumos, enquanto, indiretamente, permite flexibilizar o uso dos fatores e alcançar a escala eficiente de produção. Neste estudo, dada a multiplicidade dos efeitos dessa variável sobre a produção, optou-se por incluí-la como fonte de ineficiência técnica.

A energia, devido à modernização da agricultura nos últimos 30 anos, passou a ser um insumo essencial para a eficiência técnica de produção e o aumento da produtividade agrícola. Trabalhos investigando o consumo de energia como fonte de ineficiência técnica na agricultura são praticamente inexistentes.

A equação que expressa a ineficiência técnica, $u_{i t}$, ficou, assim, especificada: 
Em que: CREDITO $_{i t}$ é o valor do montante de empréstimos, em milhões de dólares, fornecidos pelo setor bancário privado/comercial a produtores na agricultura, silvicultura e pesca, incluindo produtores domésticos, cooperativas e agronegócios; ENERGIA é o consumo total de energia na agricultura, em terajoule; TEMPO é uma variável de tendência, que captura os efeitos ao longo do tempo; e $w_{i t}$ é o termo de erro aleatório que tem uma distribuição normal com média zero e variância constante, $N \sim\left(0, \sigma^{2}\right)$.

A variável tempo foi incluída nos modelos com finalidade de avaliar a tendência temporal da produção e da ineficiência, como sugerida por Battese \& Coelli (1995) e Araújo \& Araújo (2016). Como dito anteriormente, os parâmetros $\beta^{\prime}$ S, na Equação 5, e $\delta^{\prime}$ S, na Equação 6, são estimados simultaneamente por meio do estimador de Máxima-Verossimilhança.

\subsection{Testes de Hipóteses}

Dois testes de hipóteses são realizados a fim de selecionar o melhor modelo para análise dos dados: (i) teste da forma funcional e (ii) ausência de efeito da ineficiência. Ambos os testes utilizam a estatística de verossimilhança generalizada, que é expressa pela seguinte equação:

$L R=-2\left(\ln L L H_{0}-\ln L L H_{1}\right) \sim \chi^{2}$

Em que: $L R$ é a estatística da verossimilhança generalizada; $\ln L L H_{0}$ é o valor log-verossimilhança da hipótese nula ( $\left.H_{0}\right)$; e $\ln L L H_{0}$ é o valor do log-verossimilhança da hipótese alternativa $\left(H_{1}\right)$. O valor crítico de $L R$ é mostrado pela Tabela Kodde \& Palm (1986).

O primeiro teste busca identificar qual é a melhor forma funcional, Cobb-Douglas ou Translog, para representar os dados. Esse teste tem como hipótese nula $H_{0}$ que a primeira função Cobb-Douglas é a mais adequada para ajudar os dados. Desta forma, se o valor calculado da estatística do teste for maior que o valor crítico da Tabela Kodde \& Palm (1986), rejeita-se $H_{0}$ (i.e., a função Translog é mais conveniente).

O segundo teste consiste em verificar a inexistência da ineficiência técnica, ou seja, se de fato o modelo capta a ineficiência da unidade tomadora de decisão. Para isto, primeiro, estima-se uma função de produção com o estimador de Mínimos Quadrados Ordinários (MQO), isto é, ignorando a ineficiência no modelo. Em seguida, estima-se a função de produção estocástica, em que a ineficiência é considerada.

O teste do efeito de ineficiência técnica consiste em comparar os dois modelos, considerando as seguintes hipóteses: $H_{0}: \gamma=0$ (ausência de ineficiência) e $H_{1}: \gamma \neq 0$ (ineficiência presente). Se o valor calculado da estatística do teste for maior que o valor crítico da Tabela Kodde \& Palm (1986), rejeita-se $H_{0}$ (i.e., as UTDs possuem ineficiência técnica).

\subsection{Produtividade total dos fatores e índice de Malmquist}

Este estudo utiliza o índice de Malquist, que é obtido pela relação entre o índice agregado de produto e o índice agregado de insumos. O índice de Malmquist permite descrever uma tecnologia de produção por meio das funções distâncias, ou seja, sem a necessidade de especificar uma função objetivo comportamental.

Este índice é uma das abordagens mais utilizadas na literatura para mensurar a mudança na PTF, tendo sido aplicado por Marinho \& Carvalho (2004), Gomes et al. (2013), Araújo \& Mancal (2015), Araújo \& Araújo (2016) e Morais et al. (2016).

A mudança na PTF pode ser feita sempre que dados de insumos e produtos estão disponíveis para dois períodos de tempo. Considerando a orientação do produto e dois períodos de tempo, s e $t$, a mudança da PTF de Malmquist entre esses períodos, tendo o período $t$ como referência, pode ser calculada como (Coelli et al., 1998) propõem: 
$m_{0}^{t}\left(y_{t}, x_{t}, y_{s}, x_{s}\right)=\frac{d_{0}^{t}\left(y_{t}, x_{t}\right)}{d_{0}^{t}\left(y_{s}, x_{s}\right)}$.

Se a tecnologia do período $s$ for tida como referência, essa mudança será dada por:

$m_{0}^{s}\left(y_{t}, x_{t}, y_{s}, x_{s}\right)=\frac{d_{0}^{s}\left(y_{t}, x_{t}\right)}{d_{0}^{s}\left(y_{s}, x_{s}\right)}$.

Uma vez que o índice de Malmquist pode ser calculado tanto com relação ao período $s$ quanto com relação ao período $t$, no sentido de evitar a escolha arbitrária da tecnologia de referência, uma maneira de contornar esse problema é utilizar a média geométrica desses dois índices, assim especificada: $m_{0}\left(y_{t}, x_{t}, y_{s}, x_{s}\right)=\left[m_{0}^{t}\left(y_{t}, x_{t}, y_{s}, x_{s}\right) \times m_{0}^{s}\left(y_{t}, x_{t}, y_{s}, x_{s}\right)\right]^{0.5} \quad$ (Coelli et al., 1998); note-se que a média geométrica também pode ser expressa em termos das respectivas funções distâncias:

$m_{0}\left(y_{t}, x_{t}, y_{s}, x_{s}\right)=\left[\frac{d_{0}^{s}\left(y_{t}, x_{t}\right)}{d_{0}^{s}\left(y_{s}, x_{s}\right)} \times \frac{d_{0}^{t}\left(y_{t}, x_{t}\right)}{d_{0}^{t}\left(y_{s}, x_{s}\right)}\right]^{0.5}$.

Como a Equação 10 refere-se a uma medida de variação, o valor de $m_{0}\left(y_{t}, x_{t}, y_{s}, x_{s}\right)$ maior do que 1 revela uma variação positiva entre os períodos $s$ e $t$, enquanto $m_{0}\left(y_{t}, x_{t}, y_{s}, x_{s}\right)$ menor do que 1 indica que ocorreu variação negativa.

Depois de manipulações algébricas, a Equação 10 pode ser representada equivalentemente por:

$m_{0}\left(y_{t}, x_{t}, y_{s}, x_{s}\right)=\frac{d_{0}^{t}\left(y_{t}, x_{t}\right)}{d_{0}^{s}\left(y_{s}, x_{s}\right)}\left[\frac{d_{0}^{s}\left(y_{t}, x_{t}\right)}{d_{0}^{t}\left(y_{t}, x_{t}\right)} \times \frac{d_{0}^{s}\left(y_{s}, x_{s}\right)}{d_{0}^{t}\left(y_{s}, x_{s}\right)}\right]^{0.5}$,

Em que:

Variação na Eficiência Técnica $(V E T)=\frac{d_{0}^{t}\left(y_{t}, x_{t}\right)}{d_{0}^{s}\left(y_{s}, x_{s}\right)}$

e

Variação Tecnológica $(V T)=\left[\frac{d_{0}^{s}\left(y_{t}, x_{t}\right)}{d_{0}^{t}\left(y_{t}, x_{t}\right)} \times \frac{d_{0}^{s}\left(y_{s}, x_{s}\right)}{d_{0}^{t}\left(y_{s}, x_{s}\right)}\right]^{0.5}$.

Desta forma, o índice de PTF de Malmquist pode ser decomposto em duas partes: (i) o índice de variação na eficiência técnica e (ii) o índice de variação tecnológica. A decomposição do índice de Malmquist permite identificar qual desses dois índices tem maior contribuição no crescimento da produtividade total dos fatores.

A Figura 1 mostra, graficamente, a decomposição do índice de Malmquist para uma tecnologia relacionando um produto e um insumo, e sob retornos constantes de escala. No gráfico, os pontos $D$ e E representam a mesma firma produzindo em dois períodos no tempo, s e t, respectivamente. Em cada período, a firma está situada abaixo da fronteira de eficiência, ou seja, operando sob condição de ineficiente em ambos os períodos. 


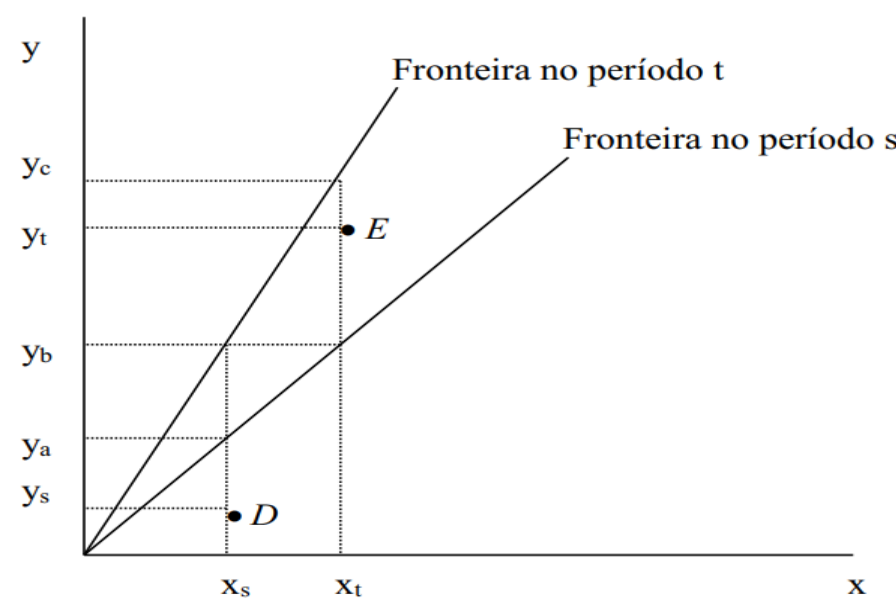

Figura 1- Índice de Produtividade de Malmquist. Fonte: Adaptado de Coelli et al. (1998).

Usando as Equações 12 e 13, os dois índices variações podem ser obtidos, ou seja:

Variação na Eficiência Técnica $(V E T)=\frac{y_{t} / y_{c}}{y_{s} / y_{a}}$

Variação Tecnológica $(V T)=\left[\frac{y_{t} / y_{b}}{y_{t} / y_{c}} \times \frac{y_{s} / y_{a}}{y_{s} / y_{b}}\right]^{0.5}$.

Assumindo que os valores de $y_{s}, y_{a}, y_{b}, y_{t}$ e $y_{c}$ são 1, 2, 4, 6 e 7, respectivamente, e aplicando as Fórmulas 14 e 15, obtêm-se VET $=1,7$ e VT $=1,9$. Os índices estimados revelam que ocorreram variações positivas tanto na eficiência técnica como na variação tecnológica, durante o período, sendo que este último proporcionou uma maior contribuição na variação na produtividade total dos fatores, que foi de $3,2(=1,7 \times 1,9)$.

Os cálculos das distâncias podem ser feitos através de modelos não paramétricos, como a Data Envelopment Analysis (DEA), bem como métodos paramétricos, como a Stochastic Frontier Analysis (SFA). Neste estudo, utiliza-se o método SFA, seguindo as abordagens empregadas por Marinho \& Carvalho (2004), Araújo \& Mancal (2015), Ferreira (2015) e Araújo \& Araújo (2016).

\subsection{Base de Dados}

A área de estudo abrange os principais países da América Latina e do Caribe (ALC), totalizando 18 países, a saber: Argentina, Belize, Bolívia, Brasil, Costa Rica, República Dominicana, Equador, El Salvador, Guatemala, Guiana, Jamaica, México, Nicarágua, Panamá, Peru, Suriname, Trinidad Tobago e Uruguai. Outros países da ALC não foram incluídos na análise pelo fato de o seu setor agrícola ser inexpressivo e/ou não dispor de dados para as variáveis de interesse.

Os dados do setor agropecuário dos países da ALC foram extraídos das bases de dados da Organização das Nações Unidas para Alimentação e Agricultura (FAO), das Nações Unidas (ONU) e do Departamento de Agricultura dos Estados Unidos (USDA). O período de análise abrangeu um período de 21 anos, cobrindo os anos de 1991 a 2012.

Os dados de produção da agricultura, crédito agrícola e consumo de energia foram coletados da base de dados da FAO (Food and Agriculture Organization of the United Nations, 2018), enquanto os dados de pessoal que trabalha na agricultura, área destinada à agricultura e número de máquinas agrícolas foram extraídos da base de dados da USDA (United States of Department of Agriculture, 2018). Esses dados formaram um painel desbalanceado, com 
206 observações, uma vez que nem todos os países apresentaram dados completos de crédito e consumo de energia para todo o período.

Os procedimentos computacionais e econométricos foram realizados em ambiente estatístico $R$ (R Core Team, 2017), com o auxílio de pacotes adicionais (add-on packages), tais como: o frontier (Coelli \& Henningsen, 2017), para estimar as fronteiras estocásticas, e o plm (Croissant \& Millo, 2017), para ordenar os dados em forma de painel.

\section{Resultados e Discussão}

Esta seção apresenta o sumário estatístico das variáveis que descrevem o setor agropecuário dos países da ALC e os resultados das estimações de fronteira estocástica de produção e dos índices de Malmquist.

\subsection{Estatísticas descritivas das variáveis}

A Tabela 1 apresenta o sumário estatístico das variáveis contidas no modelo de fronteira estocástica de produção, calculado de forma agregada para a América Latina e o Caribe, no período de análise (todos os países e todos os anos).

Tabela 1 - Sumário estatístico das variáveis

\begin{tabular}{cccccc} 
Variáveis & Média & Desvio padrão & Mínimo & Máximo & CV (\%) \\
PRODUÇÃO (ton) & 26.954 .370 & 76.401 .727 & 425.650 & 97.716 .209 & 283 \\
AREA (1.000 ha) & 6.783 & 13.103 & 64 & 86.658 & 193 \\
TRABALHO (1.000 pessoas) & 1.052 & 1.933 & 19 & 10.495 & 184 \\
MAQUINA (un) & 53.213 & 112.566 & 825 & 930.582 & 212 \\
ENERGIA (TJ) & 26.210 & 51.138 & 0 & 344.604 & 195 \\
CREDITO (US\$ milhões) & 853 & 1.649 & 13 & 10.429 & 193 \\
\hline
\end{tabular}

Fonte: Elaborado pelos autores. Nota: a produção (toneladas) é a soma da quantidade produzida pela pecuária (ton.) com a agricultura (ton.).

De acordo com a Tabela 1, a média da produção da agricultura foi de 26,9 milhões de toneladas, oscilando entre 425,7 mil toneladas, observada no Suriname em 2006, e 1,05 bilhão de toneladas, no Brasil em 2012. Os valores máximos de todas as variáveis referem-se ao melhor desempenho do Brasil nessas variáveis. O Suriname obteve ainda o menor valor para a variável máquina, observado no ano de 2006.

A variável área apresentou a média de 6,7 milhões de hectares, sendo o menor valor observado de 64 mil hectares, relativo à área agrícola de Trinidade Tobago, em 1998. Este país realizou ainda o menor consumo de energia, zero terajoule, em 1999. O Brasil despontou com a maior área agrícola no ano de 2012, com 86,6 milhões de hectares.

O total de pessoas ocupadas na agricultura, em média, foi de 1,05 milhão de pessoas, sendo Belize o que apresentou o menor contingente de pessoas ocupadas, 19 mil pessoas nos anos de 1991 e 1992. Já o valor médio de máquinas na agricultura foi de 53,213 unidades. O Brasil destaca-se entre os países da ALC por apresentar o maior contingente de pessoas ocupadas no setor agrícola, de 10,4 milhões de pessoas, no ano de 2012.

As médias do montante de crédito e do consumo de energia empregados na agropecuário tiveram valores de 853 milhões de dólares e 26.210 terajoules, respectivamente. A Guiana apresentou o menor valor de crédito, com 13 milhões de dólares, em 1994. O Brasil, no ano de 2012 , empregou $R \$ 10,4$ bilhões em crédito e 344,6 bilhões de terajoules no setor agropecuário, sendo estes os maiores valores observados entre os países da ALC para essas variáveis no período analisado.

Em geral, as variáveis apresentaram amplitudes totais elevadas, as quais são obtidas pela diferença entre o valor máximo e o valor mínimo de cada variável. Da mesma forma, as 
variáveis apresentaram elevadas variabilidades, tendo a produção e a máquina mostrado os maiores coeficientes de variação (CV), de $283 \%$ e $212 \%$, respectivamente.

\subsection{Fronteira estocástica de produção (SFA)}

A Tabela 2 mostra os resultados da estimação do modelo de fronteira estocástica de produção agrícola dos países da América Latina e do Caribe (ALC). As médias das estimativas dos coeficientes dos modelos de SFA, acompanhados de suas estatísticas, e das eficiências técnicas são apresentadas.

Tabela 2 - Estimação da fronteira estocástica de produção

\begin{tabular}{|c|c|c|c|c|}
\hline Variáveis & Estimativas & Desvio Padrão & Valor Z & $\operatorname{Pr}>|z|$ \\
\hline$\beta_{0}$ (Intercepto) & 11,869966 & 0,25370 & 46,7870 & 2,2e-16 \\
\hline $\ln A R E A$ & 0,364269 & 0,04491 & 8,1109 & $5,2 \mathrm{e}-16$ \\
\hline $\ln T R A B A L H O$ & 0,318304 & 0,02291 & 11,3890 & $2,2 e-16$ \\
\hline $\ln$ MAQUINA & $-0,002066$ & 0,04326 & $-0,0004$ & 0,9619 \\
\hline TEMPO & 0,080457 & 0,00138 & 40,7340 & $2,2 e-16$ \\
\hline \multicolumn{5}{|c|}{ Ineficiência } \\
\hline$\delta_{0}$ (Intercepto) & 0,528891 & 0,12495 & 4,2327 & $2,3 e-05$ \\
\hline CREDITO & $-0,000133$ & 0,00004 & $-3,3720$ & 0,0007 \\
\hline ENERGIA & $-0,000005$ & 0,000001 & $-3,6736$ & 0,0002 \\
\hline TEMPO & 0,092083 & 0,00796 & 11,5558 & $2,2 \mathrm{e}-16$ \\
\hline \multicolumn{5}{|c|}{ Variância } \\
\hline$\sigma^{2}$ & 1,9421e-01 & 0,02246 & 8,6442 & $2,2 \mathrm{e}-16$ \\
\hline$\gamma$ & 0,9999 & $9,37 e-08$ & $1,06 e+07$ & $2,2 \mathrm{e}-16$ \\
\hline$\sigma_{u}^{2}$ & 1,9421e-01 & 0,02246 & 8,6442 & $2,2 \mathrm{e}-16$ \\
\hline$\sigma_{v}^{2}$ & 1,9421e-09 & 1,82e-08 & 0,1062 & 0,9153 \\
\hline$\lambda$ & $1 e+04$ & $4,68 e+04$ & 0,2134 & 0,8310 \\
\hline ET média & 0,2729 & - & - & - \\
\hline ET máxima & 0,9998 & - & - & - \\
\hline ET mínima & 0,0373 & - & - & - \\
\hline
\end{tabular}

Fonte: Elaborado pelos autores. Nota: o número de observações é 206; a Log Verossimilhança é -110,77; a Razão de Verossimilhança Generalizada (LR teste de $u_{i t}$ ) é de 73,41 com $\mathrm{p}>\chi^{2}=0,0000$.

Vê-se na Tabela 2 que a estimativa da variável Máquina não foi estatisticamente significativa, mesmo considerando o nível de $10 \%$. Todas as outras variáveis restantes do modelo SFA obtiveram coeficientes estatisticamente significativos a 1\%. Da mesma forma, os coeficientes apresentaram os sinais positivos, portanto, com a direção esperada, o que corroboraram os resultados obtidos por Araújo et al. (2014), Helfand et al. (2015) e Ferreira et al. (2016). Esses coeficientes correspondem à própria medida de elasticidade da produção à variação no insumo.

A Área foi o insumo agrícola que apresentou a maior elasticidade de produção, 0,36, indicando que $1 \%$ de aumento nesse fator elevará o produto agrícola em $0,36 \%$, ceteris paribus. A elasticidade da produção relativa às mudanças no fator trabalho é cerca de 0,32. Esses resultados revelaram que a produção agrícola nos países da ALC mantém-se inelástica às variações nesses fatores de produção. Porém, tal resultado não é geral na ALC.

O coeficiente da tendência temporal (tempo) apresentou valor positivo, indicando que, no período analisado, ocorreu progresso técnico na produção agrícola dos países analisados. 
Esse resultado corroborou aqueles obtidos por Marinho \& Bittencourt (2007) e Araújo et al. (2014).

Ainda recorrendo à Tabela 2, o indicador de ineficiência, $\gamma$, foi estimado em 0,99, significando que $99 \%$ da variância total do erro composto na Equação 1 é explicada pela ineficiência técnica. Segundo o teste de Verossimilhança Generalizada (LR teste de $u_{i t}$ ), a hipótese de inexistência de efeitos de ineficiência técnica na função de produção foi rejeitada no nível de $1 \%$ de significância, implicando que o termo de ineficiência técnica deve ser incorporado ao modelo. Tal fato também corroborou os dados de Marinho \& Bittencourt (2007), Araújo et al. (2014) e Ferreira et al. (2016).

A ineficiência técnica foi explicada significativamente por todas as variáveis investigadas como fontes de ineficiência - o crédito, a energia e o tempo. Os coeficientes do crédito agrícola, do consumo de energia e da tendência temporal foram significativos no nível de $1 \%$ de significância.

O coeficiente do crédito apresentou sinal negativo, sendo este a direção esperada, ou seja, mantendo-se todas as variáveis constantes, o aumento no montante de crédito agrícola utilizado pelos países da ALC faz com que a ineficiência técnica nesse setor diminua. Em outras palavras, embora de baixa magnitude, a expansão no crédito agrícola está associada positivamente à elevação na eficiência técnica de produção.

Com relação ao consumo de energia, o efeito marginal desse insumo sobre a ineficiência de produção agrícola foi negativo, como era de se esperar, i.e., o aumento do uso da energia na agricultura está associado à redução da ineficiência técnica da produção. Esta relação pode ser explicada pelo fato de que as tecnologias modernas são dependentes desse insumo e podem conduzir a ganhos de produtividade.

Como dito anteriormente, a variável de tempo foi incluída como argumento do modelo de ineficiência técnica com a finalidade de capturar a sua evolução ao longo do tempo. O coeficiente da variável de tempo foi positivo e significativo. Isto mostra que o setor agrícola nos países da ALC experimentou perdas de eficiência técnica na produção. Tal resultado corroborou o achado por Araújo \& Araújo (2016).

Ainda de acordo com a Tabela 2, a eficiência média estimada (ET média) foi de 0,2729 ou $27,29 \%$. Isto significa que, em média, considerando o mesmo nível tecnológico, os países da ALC produziam $27,29 \%$ do produto agrícola obtido pelos países localizados sobre a fronteira de produção. Para alcançar a fronteira de eficiência, o produto agrícola dos países ineficientes deveria aumentar o produto em $366,4 \%$ [ $=(1 / 0,2729) * 100]$, mantendo o nível dos insumos constantes.

A Tabela 3 mostra cinco países mais eficientes e os cinco países menos eficientes, acompanhados dos valores médios do índice de eficiência técnica e das variáveis de produção e de ineficiência técnica.

Tabela 3 - Médias das variáveis para os cinco países mais e os cinco países menos eficientes

\begin{tabular}{ccccccccc}
\multirow{2}{*}{ Países } & Produção & Área & Trabalho & Máquina & Energia & Crédito & Eficiência \\
\cline { 2 - 8 } & & \multicolumn{7}{c}{ Mais Eficientes } \\
Brasil & 977.162 .097 & 86.658 & 10.495 & 930.582 & 344.604 & 10.429 & 0,9998 \\
Argentina & 103.855 .949 & 34.886 & 1.440 & 276.266 & 121.986 & 4.374 & 0,6393 \\
Belize & 1.422 .022 & 98 & 22 & 1.202 & 250 & 35 & 0,4546 \\
Costa Rica & 9.604 .922 & 602 & 322 & 7.596 & 3.211 & 357 & 0,4392 \\
Trinidad Tobago & 1.414 .302 & 67 & 50 & 4.826 & 322 & 50 & 0,3451 \\
& & \multicolumn{7}{c}{ Menos Eficientes } \\
Nicarágua & 6.138 .854 & 2.367 & 373 & 2.884 & 599 & 219 & 0,1194 \\
Peru & 29.492 .564 & 7.528 & 3.715 & 14.692 & 10.872 & 1.895 & 0,1173 \\
Bolívia & 11.631 .056 & 4.603 & 1.789 & 6.821 & 9.659 & 363 & 0,1117 \\
Panamá & 3.348 .968 & 771 & 264 & 9.386 & 840 & 596 & 0,0938 \\
Suriname & 473.689 & 99 & 33 & 1.047 & 3.150 & 25 & 0,0453 \\
\hline
\end{tabular}

Fonte: Elaborado pelos autores. 
De acordo com a Tabela 3, os cinco países com os maiores índices de eficiência técnica foram Brasil (0,9998), Argentina (0,6393), Belize $(0,4546)$, Costa Rica $(0,4392)$ e Trinidad Tobago $(0,3451)$. Desse grupo de países, Brasil e Argentina destacam-se tanto em termos de produção quanto de emprego de insumos. Porém, a maior eficiência técnica obtida pelo Brasil está apoiada no desempenho excepcional de sua produção agrícola.

Os cinco países com os menores índices de eficiência técnica foram Nicarágua $(0,1194)$, Peru $(0,1173)$, Bolívia $(0,1117)$, Panamá $(0,0938)$ e Suriname $(0,0453)$. Nesses países, a escala de produção mostrou-se consideravelmente baixa quando comparada com a dos demais países mais eficientes.

Explicar a hierarquização dos países em termos de eficiência técnica com base nas médias de produção obtida e insumos empregados é uma tarefa difícil e imprecisa. Porém, pode-se inferir com base nas suas médias que, para alguns países, certos fatores de produção podem ter funcionado como fator limitante para produção e, para outros países, tais fatores podem ser caracterizados como uso perdulário. De tal forma que, conjuntamente, essas relações podem fazer declinar a eficiência de um país relativamente a outro. Porém, em geral, observa-se que os países que apresentaram maiores valores para as variáveis de energia e crédito possuíam os maiores índices de eficiência técnica. Isso pode ser um indício de que essas variáveis contribuíram para a redução da ineficiência técnica de produção agrícola na América Latina e no Caribe.

\subsection{Produtividade total dos fatores (PTF)}

As estimativas da variação da produtividade total dos fatores (PTF) e de seus componentes, variações da eficiência técnica (VET) e tecnológica (VT), são apresentadas na Tabela 4.

Tabela 4 - Decomposição da produtividade total dos fatores - PTF (índices cumulativos)

\begin{tabular}{cccc} 
Ano & VET & VT & PTF \\
1991 & 1,0000 & 1,0000 & 1,0000 \\
1992 & 0,8836 & 0,9998 & 0,8834 \\
1993 & 0,7748 & 0,9498 & 0,7359 \\
1994 & 0,6681 & 0,8834 & 0,5902 \\
1995 & 0,5745 & 0,8309 & 0,4774 \\
1996 & 0,4845 & 0,7924 & 0,3839 \\
1997 & 0,4077 & 0,7277 & 0,2957 \\
1998 & 0,3429 & 0,7197 & 0,2468 \\
1999 & 0,2848 & 0,7174 & 0,2043 \\
2000 & 0,2376 & 0,7349 & 0,1746 \\
2001 & 0,1957 & 0,7230 & 0,1415 \\
2002 & 0,1605 & 0,7212 & 0,1157 \\
2003 & 0,1325 & 0,7289 & 0,0966 \\
2004 & 0,1089 & 0,7396 & 0,0805 \\
2005 & 0,0894 & 0,7807 & 0,0698 \\
2006 & 0,0723 & 0,7855 & 0,0568 \\
2007 & 0,0582 & 0,8298 & 0,0483 \\
2008 & 0,0467 & 0,9131 & 0,0427 \\
2009 & 0,0370 & 1,0257 & 0,0379 \\
2010 & 0,0292 & 1,1008 & 0,0322 \\
2011 & 0,0229 & 1,2049 & 0,0276 \\
2012 & 0,0188 & 1,6652 & 0,0313 \\
\hline
\end{tabular}

Fonte: Elaborado pelos autores. 
De acordo com a PTF, o setor agrícola dos países da ALC experimentou decrescimento da produtividade durante todo o período analisado. A VET também apresentou o mesmo comportamento.

Entre o período de 1992 a 2008, os índices de variação do progresso técnico ou variação tecnológica apresentaram resultados inferiores à unidade, o que evidencia declínio do padrão tecnológico nos países da ALC e implica no deslocamento da fronteira de produção do bloco para baixo. Já entre 2009 e 2012, a VT denotou variação positiva da fronteira de produção. De 1991 a 2012, a VT denotou aumento de 66,52\%.

Em termos da validade dos resultados obtidos nesta seção, a literatura mostra-se ambígua. Corroborando os resultados deste estudo, Morais et al. (2016), ao analisarem os países do Mercosul, entre 1991 e 2010, mostraram que ambos os grupos de países, membros e não membros do MERCOSUL, apresentaram queda da PFT da ordem de 6,6\% e 5,17\%, respectivamente. Da mesma forma, os autores constataram que a VET e a VT declinaram durante o período.

Marinho \& Bittencourt (2007) mostraram que o progresso técnico nos países da América Latina foi o principal responsável pelo crescimento da PTF. Contudo, aqui, apesar de a VT apresentar crescimento nos últimos quatro anos, tal fato não fez com que a PTF se elevasse, uma vez que o declínio da VET foi mais acentuado. Alinhados com Marinho \& Bittencourt (2007), Araújo et al. (2014) e Ferreira et al. (2016) concluíram que a América Latina apresentou ganhos de produtividade total dos fatores, sendo o maior responsável por tal façanha o progresso tecnológico ocorrido nos países latino-americanos.

Com base nos resultados obtidos pelo índice de produtividade de Malmquist, nota-se que não houve aumento da produtividade total dos fatores, resultado que corroborou os achados de Hutchinson (2007), Zúniga-González (2011) e Trindade \& Fulginiti (2015), mas que discordou de Ludena (2010).

\section{Considerações Finais}

Este artigo avaliou a eficiência técnica e a mudança na produtividade total dos fatores, e de seus componentes, do setor agrícola de 18 países da América Latina e do Caribe (ALC), no período de 1991 a 2012. Utilizando o modelo de fronteira estocástica de produção, a variabilidade do produto agropecuário foi explicada pelos fatores terra, capital e trabalho, e pelas fontes de ineficiência técnica atribuídas ao consumo de energia e ao crédito agrícola.

Os resultados mostraram que as variáveis de insumos (área, trabalho) contribuíram para a expansão do produto agropecuário dos países da ALC. A variável máquina apresentou sinal negativo, porém não foi estatisticamente significativa sequer no nível de $10 \%$.

O consumo de energia e o montante de crédito agrícola explicaram significativamente a ineficiência técnica do setor agropecuário dos países da ALC, de tal forma que aumentos nesses insumos contribuíram para diminuir a ineficiência técnica da produção. Este resultado tem sua relevância para o setor agrícola, pois demonstra que o aumento na disponibilidade de crédito agrícola, associado ao emprego de tecnologia moderna que aumente o consumo de energia, pode levar ao aumento da eficiência da produção agrícola.

Com relação à produtividade total dos fatores, medida pelo índice de Malmquist, viu-se que, em todo o período analisado, os países não apresentaram ganhos de produtividade. A variação da eficiência técnica apresentou declínio em todo o período de análise. Assim, mesmo ocorrendo crescimento da variação tecnológica nos últimos quatro anos, a PTF não se elevou, uma vez que a redução da VET é mais acentuada que o aumento da VT. Porém, deve-se ressaltar que a literatura ainda é ambígua com relação às mudanças técnicas e tecnológicas no setor agropecuário dos países da ALC, o que aponta para a necessidade do aprofundamento da investigação sobre esse tema.

\section{Agradecimentos}

Os autores agradecem à Coordenação de Aperfeiçoamento de Pessoal de Nível Superior (CAPES/MEC) pela bolsa concedida. 


\section{Referências}

Aigner, D. J., Lovell, C. A. K., \& Schmidt, P. (1977). Formulation and estimation of stochastic frontier production functions models. Journal of Econometrics, 6, 21-37.

Araújo, J. A., \& Mancal, A. (2015). Produtividade e eficiência no setor agropecuário do Nordeste brasileiro. Interações, 16(2), 385-394.

Araújo, J. A., Feitosa, D. G., \& Silva, A. B. (2014). América Latina: productividad total de los factores y su descomposición. Revista de la CEPAL (Online), 114, 31-52.

Araújo, W. B. C., \& Araújo, J. A. (2016). Produtividade, variação da eficiência técnica e tecnológica na agricultura dos municípios cearenses. Interações, 17(2), 223-233.

Battese, G. E., \& Coelli, T. J. (1995). A model for technical inefficiency effects in stochastic frontier production functions for panel data. Empirical Economics, 20, 325-332.

Battese, G. E., \& Corra, G. S. (1977). Estimation of a production frontier model: with aplication to the pastoral zone of Eastern Austrália. Australian Journal of Agricultural Economics, 21, 169-179.

Battese, G. E., Rao, D. S. P., \& O'Donnell, C. J. (2004). A metafrontier production function for estimation of technical efficiencies and technology gaps for firms operating under diferente technologies. Journal of Productivity Analysis, 21(1), 91-103.

Brasil. Ministério do Desenvolvimento, Indústria e Comércio Exterior - MDIC. (2014). Recuperado em 22 de novembro de 2018, de http://www.mdic.gov.br

Carvalho, R. M., \& Marinho, E. L. L. (2003). Transformações estruturais, variações na eficiência técnica e produtividade total dos fatores no setor agrícola dos países sul-americanos - 1970 a 2000. In Anais do XXXI Encontro Nacional de Economia. Porto Seguro: Encontro Nacional de Economia-ANPEC.

Coelli, T. J., Rao, D. S. P., O’Donnell, C. J., \& Battese, G. E. (1998). An introduction to efficiency and productivity analysis. Kluwer Academic Publishers.

Coelli, T., \& Henningsen, A. (2017). Frontier: Stochastic Frontier Analysis. R package version 1.1-2. Recuperado em 17 de agosto de 2018, de https://CRAN.R-Project.org/package=frontier

Croissant, Y., \& Millo, G. (2017). PLM: Linear Models for Panel Data. R package version 1.6-6. Recuperado em 17 de agosto de 2018, de https://CRAN.R-project.org/package=plm

Ebata, A. (2011). Agricultural Productivity Growth in Central America and the Caribbean (Master's thesis). University of Nebraska, Nebraska.

Ferreira, C. B. (2015). Ensaios sobre produtividade e eficiência agrícola na América Latina, no Brasil e no Vale do São Francisco (Dissertação de mestrado). Universidade Federal do Ceará, Fortaleza.

Ferreira, C. B., Araújo, J. A., Tabosa, F. J. S., \& Lima, J. R. F. (2016). Produtividade agrícola nos países da América Latina. Revista de Economia e Sociologia Rural, 54(3), 437-458.

Food and Agriculture Organization of the United Nations - FAO. (2018). FAOSTAT. Recuperado em 17 de agosto de 2018, de http://www.fao.org/faostat/en

Gomes, A. P., Alcantara Filho, J. L., \& Scalco, P. R. (2013). Mudanças recentes na estrutura de produção agropecuária do Nordeste. Revista Economica do Nordeste, 44(2), 489-506.

Helfand, S. M., Magalhaes, M. M., \& Rada, N. E. (2015) Brazil's agricultural total factor productivity growth by farm size (No. 309, IDB Working paper series). Washington: Inter-American Development Bank, Environment, Rural Development and Disaster Risk Management Division.

Hutchinson, S. D. (2007). Agricultural productivity changes in the Caribbean: challenges for trade. In Annual Meeting of American Agricultural Economics Association. Portland: 2007 Annual Meeting.

Inter-American Development Bank - IADB. (2014). America Latina frente a la desigualdad. Washington: Inter-American Development Bank.

Kodde, D. A., \& Palm, F. C. (1986). Wald criteria for jointly testing equality and inequality restrictions. Econometrica, 54(5), 1243-1248.

Ludena, C. E. (2010). Agricultural productivity growth, effciency change and technical progress in Latin America and the Caribbean (No. 186, IDB Working paper). Washington: Inter-American Development Bank.

Marinho, E., \& Bittencourt, A. (2007). Produtividade e crescimento econômico na américa latina: a abordagem da fronteira de produção estocástica. Estudos Econômicos, 37(1), 5-33.

Marinho, E., \& Carvalho, R. M. (2004). Comparações inter-regionais da produtividade da agricultura brasileira - 1970 a 1995. Pesquisa e Planejamento Economico, 34(1), 57-92. 
Meeusen, W., \& Broeck, V. D. (1977). Efficiency estimation from cobb-douglas production with composed error. Empirical Economics, 32, 715-723.

Montoya, M. A. (2002). O Agronegócio no Mercosul: dimensão econômica, desenvolvimento Industrial e interdependência estrutural na Argentina, Brasil, Chile e Uruguai. Revista Brasileira de Economia, $56(4), 605-660$.

Morais, G. A. S., Costa, E. M., Araújo, J. A., Tabosa, F. J., \& Costa, R. A. (2016). Eficiência técnica e produtividade de total dos fatores da agricultura dos países da América do Sul: uma análise dos países membros e não-membros do Mercosul. Revista de Ciências da Administração, 22(2), 396-422.

Moreno-Moreno, J., J., Sanz-Díaz, M. T. , Velasco-Morente, F., \& Ludena, C. E. (2017). A DEA-based evalution of Latin America and the Caribbean agricultural. environmental performance under the assumption of natural and managerial efficiency. Revista de Economía Mundial, 47, 157-178.

O'Donnell, C. J., Rao, D. S. P., \& Battese, G. E. (2008). Metafrontier frameworks for the study of firm-level efficiencies and technology ratios. Empirical Economics, 34(2), 231-255.

R Core Team. (2017). R: a language and environment for statistical computing. Vienna, Áustria: R Core Team. Recuperado em 15 de agosto de 2018, de https://www.R-project.org/

Scherer, C. E. M., \& Porsse, A. A. (2017). Eficiência Produtiva Regional da Agricultura Brasileira: uma análise de fronteira estocástica. Revista de Economia e Sociologia Rural, 55(2), 389-410.

Trindade, F. J., \& Fulginiti, L. E. (2015). Is there a slowdown in agricultural productivity growth in South America? Agricultural Economics, 46, 69-81.

United States of Department of Agriculture - USDA. (2018). Recuperado em 15 de agosto de 2018, de https://www.usda.gov.

Zúniga-González, C. A. (2011). Total factor productivity growth in agriculture: a malmquist index analysis of 14 countries, 1979-2008. Leon: National Autonomous University of Nicaragua.

Submissão: 2/fev./2019.

Aceito: $26 / \mathrm{Jan} . / 2020$

Classificação JEL: CO1; Q1. 